\title{
Smooth grafts ahead
}

\author{
By Kai-Jye Lou, Staff Writer
}

Researchers at the Georgia Institute of Technology have developed an approach to address a major problem with synthetic tissue grafts: poor integration with host tissue. The group used polymer-immobilized retroviruses to control cell differentiation and distribution within a graft's scaffold. The strategy potentially can be used to create interfaces between graft and host that mimic the natural transitions in musculoskeletal tissues, thus encouraging host cells to migrate into and take hold of the graft. ${ }^{1}$

Companies in the regenerative medicine space now want to see additional in vivo studies on the long-term safety and stability of grafts produced with the approach.

As published in the Proceedings of the National Academy of Sciences by Andrés García, professor of mechanical engineering at Georgia Tech's Petit Institute for Bioengineering and Bioscience, the approach involves seeding fibroblasts onto a $3 \mathrm{D}$ collagen scaffold coated with spatial gradients of polymer-immobilized retroviruses that have

"This gene-delivery strategy can also be exploited with safer delivery vectors such as lentiviruses."

- Andrés García,

Georgia Institute of Technology

osteoblasts. These transitional zones are important for proper musculoskeletal functioning at interfaces between soft tissue and bone because it allows mechanical stressors like weight, pressure and sudden acceleration to be more evenly distributed between tissue and bone.

According to García, current strategies to engineer tissue-graft interfaces use multiple cell types in multilayered 3D scaffolds. These layered constructs have discontinuous interfaces, resulting in sudden transitions from one cell type to another, he said. "That's not very robust" for transferring mechanical stress.

García told SciBX that his group's gradual transition from one cell type to another at the interface zone should allow for more efficient transfer of mechanical stress.

He added that other shortcomings of existing processes include inadequate host tissue integration, high cost, procedural complexity and performance variability. ${ }^{2-4}$

Helen Lu, director of the Biomaterials and Interface Tissue Engineering Laboratory and an associate professor of biomedical engineering at Columbia University, also thinks graded interfaces can minimize the formation of areas with high concentrations of mechanical stress and will allow for a more efficient and gradual dissipation of these stressors. "This will reduce the possibility of mechanical failure," she said.

Thus, said Lu, the PNAS paper "offers an innovative approach to interface tissue engineering, which is critical for bridging different tissue types and will certainly have clinical application in ligament-bone or tendon-bone integration." been genetically engineered to encode the osteogenic transcription factor Runx2. In situ transfection of the fibroblasts causes them to differentiate into osteoblast-like cells with the levels of differentiation being determined by the local retroviral titers.

García's research team initially focused on the soft tissue-to-bone interface because of the high number of orthopedic grafts performed annually in the U.S.

The team first coated a collagen 3D scaffold with gradually varying amounts of the polymer poly-L-lysine (PLL). The scaffold was then incubated with Runx 2 retrovirus, resulting in a structure with immobilized retrovirus loads mirroring the underlying PLL concentrations.

Next, fibroblasts were seeded onto the scaffold and transfection of Runx2 from the retroviruses was allowed to proceed. The result was a scaffold containing both fibroblasts and osteoblast-like cells where areas with higher concentrations of polymer-immobilized virus corresponded to higher numbers of osteoblast-like cells than areas with lower viral titers. $^{1}$

This ability to precisely control cell distribution patterns allows for the creation of interfaces between graft and host that have features similar to native tissue, thus encouraging host cells to take hold of the graft.

"In our strategy, we use only one cell type, one scaffold, and we find that we can generate a tissue gradient" in the graft, said García.

Tissue gradients are areas where there is a smooth, continuous transition from one cell type to another-in this case from fibroblasts to
"The strategy is very compelling," said Lonnie Shea, a professor of chemical and biological engineering at Northwestern University. He noted that many host tissues contain more than one cell type and strategies to recreate this heterogeneous distribution of cells are needed. A graded deposition of retrovirus is a clever way to get a graded distribution of cells with different functions when starting with a single cell type, he said.

"The creation of a bone-cartilage interface would be huge, considering the number of orthopedic surgeries performed on an annual basis," added Shea.

According to a paper published in the 2007 Annual Review of Biomedical Engineering, about 200,000 anterior cruciate ligament (ACL) surgeries are performed each year in the U.S., most of which require a soft-tissue graft. ${ }^{2}$

Bruce Wentworth, senior scientific director at Genzyme Corp., thinks the approach in the PNAS article could lead to the creation of tissue constructs with more cell types and more complex cell distribution patterns that closely mimic native tissue.

"It has the advantage that both the matrix and linked retrovirus should be stable and thus amenable to 'off-the-shelf' use" and thus require minimal preparation time. The simple manufacturing process for the matrices should be easy to control and is likely to produce consistent results, he told SciBX. 
Genzyme's tissue engineering products include Carticel autologous cultured chondrocytes for cartilage repair and Epicel, an epidermal autograft consisting of human cells cultured with mouse fibroblasts and bovine serum to treat burns.

\section{The rap on retroviruses}

From a regulatory standpoint, however, Wentworth was concerned by the retrovirus-based approach. "As they are integrating viruses, a longer and potentially more complex analysis of safety" compared to nonretroviral methods might be required by regulators, he said.

Wentworth expects that nonretroviral approaches are less likely to provoke safety considerations. Shea agreed and said subsequent experiments will need to demonstrate the safety of retroviral gene insertion and assess immunogenicity of constructs created using García's approach.

García agreed that the use of retroviruses brings up safety issues such as lack of target specificity and mutagenesis but noted that he believes "that this gene-delivery strategy can also be exploited with safer delivery vectors such as lentiviruses."

Retroviruses aside, Tom Van de Putte, head of technology development at TiGenix N.V., wanted to see additional work to assess construct integration with native tissue and long-term stability.

"Naturally occurring repair processes go through a consecutive and well-defined transient expression of a series of growth factors, which is basically a recapitulation of the events that occur during embryogenesis. Mimicking this by the expression of one or a few growth factors will require extensive fine tuning," he said.

TiGenix submitted a marketing authorization application to the EMEA in June 2007 for ChondroCelect, a cell-based therapy for carti- lage repair. The company expects a Committee for Medicinal Products for Human Use recommendation in $4 \mathrm{Q} 08$ and plans to submit a BLA to the FDA afterward.

Regarding safety, Van de Putte told SciBX he wanted to see additional studies on the longterm effects of continuous exposure to growth factors of the applied cells and surrounding native tissue.

García said his group is gearing up to create either bone-to-ligament or bone-to-tendon anchorage interfaces. Next steps will include experiments to compare the biomechanical properties of constructs with graded interfaces to those with discontinuous or no interface and studies to asses how host body cells respond to the gradedtissue construct. "Large animal experiments are required as well," he added.

The researchers declined to disclose the patent and licensing status of the work described in the paper.

\section{REFERENCES}

1. Phillips, J.E. et al. Proc. Natl. Acad. Sci. USA; published online Aug. 11, 2008; doi:10.1073/pnas.0801988105

Contact: Andrés J. García, Georgia Institute of Technology,

Atlanta, Ga.

e-mail: andres.garcia@me.gatech.edu

2. Gersbach, C.A. et al. Annu. Rev. Biomed. Eng. 9, 87-119 (2007)

3. Mikos, A.G. et al. Tissue Eng. 12, 3307-3339 (2007)

4. Phillips, J.E. et al. Biomaterials 28, 211-229 (2007)

COMPANIES AND INSTITUTIONS MENTIONED

Columbia University, New York, N.Y.

Genzyme Corp. (NASDAQ:GENZ), Cambridge, Mass.

Georgia Institute of Technology, Atlanta, Ga.

Northwestern University, Evanston, III.

TiGenix N.V. (Euronext:TIG), Haasrode, Belgium 